wildlife reservoirs is increasing, ${ }^{4,5}$ larger and more frequent outbreaks are to be expected. Moreover, because of the lack of awareness about monkeypox, health care-related transmission of the virus may occur. Mortality due to monkeypox can be as high as 10\% among those who are infected ${ }^{5}$ and can be particularly high among children and possibly among immunodeficient persons. There is no established treatment for monkeypox. ${ }^{3}$ Therefore, we propose that tecovirimat be provided on a compassionate basis for monkeypox virus infections in humans.

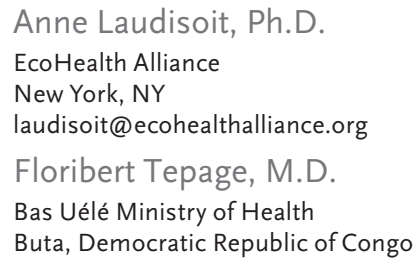

Robert Colebunders, M.D.

Global Health Institute

Antwerp, Belgium

No potential conflict of interest relevant to this letter was reported.

1. Grosenbach DW, Honeychurch K, Rose EA, et al. Oral tecovirimat for the treatment of smallpox. N Engl J Med 2018;379: 44-53.

2. Doshi RH, Guagliardo SAJ, Dzabatou-Babeaux A, et al. Strengthening of surveillance during monkeypox outbreak, Republic of the Congo, 2017. Emerg Infect Dis 2018;24:1158-60.

3. Durski KN, McCollum AM, Nakazawa Y, et al. Emergence of monkeypox - West and Central Africa, 1970-2017. MMWR Morb Mortal Wkly Rep 2018;67:306-10.

4. Hutson CL, Nakazawa YJ, Self J, et al. Laboratory investigations of african pouched rats (Cricetomys gambianus) as a potential reservoir host species for monkeypox virus. PLoS Negl Trop Dis 2015;9(10):e0004013.

5. Chen N, Li G, Liszewski MK, et al. Virulence differences between monkeypox virus isolates from West Africa and the Congo basin. Virology 2005;340:46-63.

DOI: $10.1056 / N E J M c 1811044$

\title{
Oligonucleotide Drugs for Transthyretin Amyloidosis
}

TO THE EDITOR: In his editorial describing the NEURO-TTR trial by Benson et al. ${ }^{1}$ and the related APOLLO trial by Adams et al., ${ }^{2}$ Buxbaum $^{3}$ (July 5 issue) provides a historical and biologic perspective on antisense (inotersen) and RNA interference (patisiran) therapies targeting the gene encoding transthyretin (TTR). He also writes that a post hoc test showed a correlation between a change in serum levels of transthyretin and the modified Neuropathy Impairment Score+7 (mNIS+7) only in patients who received patisiran and not in patients who received inotersen. This analysis included data from both the patients who received placebo and those who received patisiran. In contrast, the analysis by Benson et al., which included only data from patients who received inotersen, showed no correlation.

In a Pearson regression analysis, when we included data from patients who received placebo with data from patients who received inotersen, we did observe a correlation (Pearson's $r=0.35$ ); this supports the hypothesis that a reduction in transthyretin levels slows disease progression. We also note that Buxbaum's assessment of "response to any treatment" was limited to patients with neurologic function that improved by the end of the two trials. In trials of small-molecule transthyretin tetramer stabilizers, ${ }^{4,5}$ the determination of drug response was predicated on achieving less neurologic decline in patients assigned to the drug than in those assigned to placebo. By this definition, a majority of patients (approximately 70\%) benefitted from treatment with either inotersen or patisiran.

John L. Berk, M.D.

Boston University School of Medicine

Boston, MA

jberk@bu.edu

Fabio A. Barroso, M.D.

Institute for Neurological Research Raúl Carrea

Buenos Aires, Argentina

Teresa Coelho, M.D.

Hospital de Santo António

Porto, Portugal

Dr. Berk reports receiving travel support and consulting fees from Alnylam Pharmaceuticals and Ionis Pharmaceuticals; Dr. Barroso, receiving travel support and consulting fees from Ionis Pharmaceuticals and Pfizer and travel support, consulting fees, and grant support from Alnylam Pharmaceuticals; and Dr. Coelho, receiving travel support and consulting fees from Alnylam Pharmaceuticals, Ionis Pharmaceuticals, Pfizer, and GlaxoSmithKline. No other potential conflict of interest relevant to this letter was reported.

1. Benson MD, Waddington-Cruz M, Berk JL, et al. Inotersen treatment for patients with hereditary transthyretin amyloidosis. N Engl J Med 2018;379:22-31.

2. Adams D, Gonzalez-Duarte A, O'Riordan WD, et al. Patisiran, an RNAi therapeutic, for hereditary transthyretin amyloidosis. N Engl J Med 2018;379:11-21.

3. Buxbaum JN. Oligonucleotide drugs for transthyretin amyloidosis. N Engl J Med 2018;379:82-5. 
4. Berk JL, Suhr OB, Obici L, et al. Repurposing diflunisal for familial amyloid polyneuropathy: a randomized clinical trial. JAMA 2013;310:2658-67.

5. Coelho T, Maia LF, Martins da Silva A, et al. Tafamidis for transthyretin familial amyloid polyneuropathy: a randomized, controlled trial. Neurology 2012;79:785-92.

DOI: $10.1056 /$ NEJMc1810994

TO THE EDITOR: In his editorial, Buxbaum wrote that the trials that we and Benson et al. reported on were similar in design and outcome measures. However, APOLLO was unique in that it showed benefits of patisiran on prespecified hierarchically tested secondary end points that assessed motor strength, disability, gait, modified bodymass index, and autonomic symptoms. Also unique to the trial was an analysis involving the subgroup of patients with cardiac involvement, the results of which suggested a treatment effect on cardiac structure and function. In response to Buxbaum's statement that "at best only 56\% of participants had a response to any treatment," we would respectfully point out that in a prespecified threshold analysis, we observed improvement relative to baseline impairment (according to the mNIS+7) in $56 \%$ of patients who received patisiran versus $4 \%$ of those who received placebo (see Fig. 2D of our article, available at NEJM.org).

A post hoc analysis involving patients who were randomly assigned to receive patisiran and who did not have improvement relative to baseline supported less progression of neuropathy in these patients than in those who received placebo (see Fig. S1 in the Supplementary Appendix, available with the full text of this letter at NEJM.org). These findings indicate a treatment effect in the majority of patients.

\section{David Adams, M.D., Ph.D. \\ Assistance Publique-Hôpitaux de Paris Le Kremlin-Bicêtre, France david.adams@aphp.fr \\ Philip N. Hawkins, F.Med.Sci. \\ University College London \\ London, United Kingdom \\ Michael Polydefkis, M.D., M.H.S. \\ Johns Hopkins Medicine \\ Baltimore, MD}

Dr. Adams reports receiving grants from Alnylam Pharmaceuticals; and Dr. Polydefkis, receiving grants, fees for serving on an advisory board, and travel fees from Alnylam Pharmaceuticals, grants, fees for serving on an advisory board, and travel fees from Ionis Pharmaceuticals, grants and travel fees from Pfizer, consulting fees from Vertex Pharmaceuticals, grants and fees for serving on an advisory board from Biogen, and fees for serving on an advisory board from Chromocell. No other potential conflict of interest relevant to this letter was reported.

DOI: 10.1056/NEJMc1810994

THE EDITORIALIST REPLIES: The articles on oligonucleotide-based therapies for transthyretinrelated polyneuropathies reflect the commitment of scientists at Ionis Pharmaceuticals and Alnylam Pharmaceuticals in bringing these compounds from biologic observations to the clinic. In my editorial, I emphasized the clinical aspects of the two trials (i.e., therapeutic efficacy and potential adverse effects). The quality of the trials and the similarity of the protocols, demographic variables, and primary end points allow the reader, while focusing on efficacy, to identify potential insights into mechanisms of action of the different therapies.

The reanalysis by Berk and colleagues of the relationship between the extent of reduction in serum transthyretin levels and clinical response yielded a Pearson correlation coefficient of 0.35 , which differs from that obtained by Adams et al. (Pearson's $r=0.59$ ). Both results indicate that the reductions in the circulating transthyretin levels were not perfectly correlated with the clinical response, as measured by the mNIS+7. It is not clear to what extent the difference in correlation coefficients is meaningful, but a true difference would be consistent with the two oligonucleotides having therapeutic effects through mechanisms in addition to or other than lowering of transthyretin levels. It remains the case that neither of the therapies nor any previously reported treatment for this disease benefitted all the recipients. An important goal now is to devise a means by which to select the best therapy for each patient.

Joel N. Buxbaum, M.D.

Scripps Research Institute

La Jolla, CA

jbux@scripps.edu

Dr. Buxbaum reports receiving fees for serving as consulting chief medical officer for Misfolding Diagnostics. An updated financial disclosure form has been posted with the full text of the editorial at NEJM.org. No further potential conflict of interest relevant to this letter was reported.

DOI: $10.1056 /$ NEJMc1810994

Correspondence Copyright $(\odot 2018$ Massachusetts Medical Society. 